\title{
Identification and validation of potential long non-coding RNA biomarkers in predicting survival of patients with head and neck squamous cell carcinoma
}

\author{
JUNYU LI ${ }^{1}$, YUEHUA LI $^{2}$, XIAOPING WU ${ }^{2}$ and YING LI ${ }^{3}$ \\ ${ }^{1}$ Department of Radiotherapy, Cancer Hospital of Jiangxi Province, Nanchang, Jiangxi 330029; \\ ${ }^{2}$ Department of Medical Oncology, The First Affiliated Hospital of University of South China, Hengyang, \\ Hunan 421001; ${ }^{3}$ Department of Radiation Oncology, Hubei Cancer Hospital, Wuhan, Hubei 430079, P.R. China
}

Received March 19, 2018; Accepted March 21, 2019

DOI: $10.3892 / \mathrm{ol} .2019 .10261$

\begin{abstract}
Long non-coding RNAs (lncRNAs) are frequently dysregulated in cancer and their aberrant expression has been associated with cancer diagnosis and prognosis, which suggests that they may be promising molecular biomarkers. However, understanding of the expression pattern of lncRNAs and their prognostic roles in head and neck squamous cell carcinoma (HNSCC) is relatively limited. In the current study, the prognostic value of lncRNA expression profiles in predicting the OS of patients with HNSCC was investigated by integrating clinical and profiling data from The Cancer Genome Atlas. A total of ten lncRNAs closely associated with the prognosis of patients with HNSCC were identified and may serve as novel biomarkers. This 10 -lncRNA signature was used to classify patients into 2 groups with significantly different overall survival (OS) times (median OS time, 1.65 vs. 13.04 years; $\mathrm{P}<0.0001)$. This lncRNA signature was validated in an independent testing cohort. The results of multivariable Cox regression and stratification analyses revealed that the prognostic value of the 10-lncRNA signature was independent of other clinical and pathological factors for the survival of patients with HNSCC. Functional analysis demonstrated that lncRNA expression-based risk scoring may reflect the basic status of the immune response in the tumor microenvironment. The presented study demonstrated the value of a lncRNA signature as a potential biomarker to improve the clinical prognosis of patients with HNSCC.
\end{abstract}

Correspondence to: Dr Ying Li, Department of Radiation Oncology, Hubei Cancer Hospital, 116 Zhuodaoquan South Road, Hongshan, Wuhan, Hubei 430079, P.R. China

E-mail: hbwhw08@163.com

Key words: long non-coding RNA, head and neck squamous cell carcinoma, survival, biomarker, signature

\section{Introduction}

Head and neck squamous cell carcinoma (HNSCC), arising in the oral cavity, oropharynx, hypopharynx and larynx, is the sixth most common type of cancer worldwide, with $\sim 635,000$ new cases diagnosed annually and $>12 \%$ of these cases occurring in China (1). Advances in early diagnosis and surgical techniques combined with radiotherapy and chemotherapy have improved the survival rate of patients with HNSCC in the last 20 years, and the overall 5 -year relative survival rate has increased from $54.7 \%$ (1992-1996) to 65.9\% (2002-2006) (2). However, even among patients with HNSCC of the same classification, the prognosis may vary (3). Therefore, there is a requirement to identify novel molecular biomarkers of aggressive tumor behavior.

The human genome encodes $\sim 20,000$ protein-coding genes, accounting for $<2 \%$ of the human genome, as the majority of the human genome is actively transcribed into non-coding RNAs (ncRNAs) (4). These ncRNAs are divided into two categories based on their sequence length: Short ncRNAs, including microRNAs (miRNAs), and long ncRNAs (lncRNAs). IncRNAs are often defined as transcripts $>200$ nucleotides in length that lack protein-coding capacity (5). IncRNAs function in regulation of gene expression and cellular activity through diverse mechanisms (6). Previous studies have suggested that lncRNA expression is frequently dysregulated in cancer and that aberrant expression is associated with cancer diagnosis and prognosis, suggesting that lncRNAs may be promising molecular biomarkers (7-10). Certain lncRNAs have been implicated in HNSCC, including H19 imprinted maternally expressed transcript (11), HOX transcript antisense RNA (12) and cytoskeleton regulator RNA (13). However, the understanding of IncRNA expression patterns and their prognostic roles in HNSCC remains limited.

The aim of the current study was to determine the prognostic value of IncRNA expression profiles and to identify novel lncRNA biomarkers closely associated with the OS of patients with HNSCC using a large cohort of $>400$ patients with HNSCC. 


\section{Materials and methods}

HNSCC dataset. The clinical features of the patients with HNSCC used in the present study were obtained from The Cancer Genome Atlas (TCGA; tcga-data.nci.nih.gov/). The lncRNA expression data were downloaded from the The Atlas of Noncoding RNAs in Cancer (bioinformatics.mdanderson. org/main/TANRIC:Overview), in which IncRNA expression was quantified and normalized using reads per kilobase per million mapped values (14). To investigate the association between IncRNA expression and OS of patients with HNSCC, only patients with available survival data and lncRNA expression profiles were selected. Thus, 425 patients were selected and randomly divided into a training cohort $(n=213)$ and testing cohort $(\mathrm{n}=212)$ for identifying and validating survival-associated lncRNA biomarkers.

Identification of survival-associated IncRNA biomarkers. Univariate Cox regression analysis was used to assess the association between IncRNA expression and OS time in the training cohort. IncRNAs achieving significance of $\mathrm{P}<0.01$ were considered as candidate survival-associated lncRNAs. These candidate survival-associated lncRNAs were then analyzed using multivariate Cox regression analysis, and those achieving $\mathrm{P}<0.01$ in this analysis were identified as independent survival-associated lncRNAs. All survival-associated IncRNAs were combined to construct a lncRNA expression signature using a risk scoring method. A lncRNA expression signature was constructed using a risk-scoring method as previously described (7,8,15-17): The lncRNA expression signature was established by including the expression values of each selected IncRNA, weighted by their estimated regression coefficients from the multivariate Cox regression analysis. A risk score was calculated for each patient using the lncRNA expression signature. Patients were further divided into low-risk and high-risk groups using the median score of all patients of the training cohort as the cut-off point.

Statistical analysis. The Kaplan-Meier method and a log-rank test were used to compare the difference in OS time between the high- and low-risk groups. Univariate and multivariate Cox regression analyses for OS time were performed for individual clinical features, with and without consideration of the lncRNA expression signature in each cohort. Hazard ratios (HR) and 95\% confidence intervals (CI) were calculated. Time-dependent receiver operating characteristic (ROC) curve analysis for the 3-year OS time was performed to assess the prognostic value of the lncRNA expression signature using the timeROC package (version 0.3) in R (18). The survival analysis, univariate and multivariate Cox regression analyses was performed using the survival package in $\mathrm{R}$ (https://github.com/therneau/survival) (19). The correlations between protein-coding genes and the lncRNA biomarkers were identified by Pearson's correlation coefficient using the entire TCGA data cohort of 425 patients with HNSCC. All statistical analyses were performed using R/Bioconductor (version 3.0.2; bioconductor.org/).

Function enrichment analysis. To investigate the potential biological role of the identified lncRNA biomarkers,
Gene Ontology (GO; http://geneontology.org/) and Kyoto Encyclopedia of Genes and Genomes (KEGG; https://www.genome.jp/kegg/) functional enrichment analysis of protein-coding genes associated with the identified lncRNA biomarkers was performed using The Database for Annotation, Visualization and Integrated Discovery (DAVID) (v.6.8; david. ncifcrf.gov/).

\section{Results}

Identification of prognostic lncRNA biomarkers in HNSCC. To identify lncRNAs associated with the OS time of patients with HNSCC, univariate Cox regression analysis was used to assess the association between lncRNA expression and OS time in the training cohort. A total of 32 lncRNAs were demonstrated to be significantly associated with OS time for patients with HNSCC $(\mathrm{P}<0.01)$ and were considered as candidate survival-associated lncRNAs (Fig. 1A). By performing multivariable Cox regression analysis on the 32 candidate survival-associated lncRNAs, 10 candidate survival-associated lncRNAs $(\mathrm{P}<0.01$; Table I) were identified as potential independent prognostic IncRNA biomarkers. A total of four prognostic lncRNAs were identified as risk factors and their overexpression was associated with a shorter OS time (Table I). The remaining six prognostic lncRNAs were protective factors and their overexpression was associated with longer OS time (Table I).

Construction and evaluation of the IncRNA expression signature in predicting survival in the training cohort. To construct an IncRNA-based prognostic model for predicting the OS time of patients with HNSCC in the training cohort, the ten IncRNA biomarkers were fitted into a multivariable Cox regression model. A lncRNA expression signature was constructed using a risk-scoring method as previously described (6,7,15-17): lncRNA-based risk score $=[(1.2680 \mathrm{x}$ expression value of AC002066.1 $)+(0.7834 \mathrm{x}$ expression value of AC002351.1) + (3.8306 $x$ expression value of LINC00968) + (7.3969 x expression value of AF213884.3) + (1.7179 $\mathrm{x}$ expression value of AC067838.1 $)+(2.7346 \mathrm{x}$ expression value of AC015911.3 $)+$ $(0.7579 x$ expression value of LINC02159) + (3.5427 x expression value of AC090948.1) + (3.5687 x expression value of AL031714.1) + (11.2941 x expression value of DLEU7-AS1)].

A patient with HNSCC was classified as low-risk $(n=106)$ if their risk score was lower than the median risk score of the training cohort (-1.0376) and as high-risk $(\mathrm{n}=107)$ if it was higher. Patients with high-risk scores exhibited poorer OS times compared with patients with low-risk scores (median OS time, 1.65 vs. 13.04 years; $\mathrm{P}<0.0001$; Fig. 1B). In univariate analysis, the hazard ratios of the high-risk group vs. the low-risk group for OS time were 5.142 (95\% CI, 2.924-9.043; $\mathrm{P}<0.0001$; Table II). The 3- and 5-year survival rates of the high-risk group were 34.8 and $16.8 \%$, respectively, whereas those of the low-risk group were 77 and $72.7 \%$, respectively. The area under the curve (AUC) of the time-dependent ROC curve for the lncRNA expression signature was 0.796 for 3 -year OS time ( $\mathrm{P}<0.01$; Fig. 1C).

Independent validation of the IncRNA expression signature in the testing and entire TCGA cohorts. To test the predictive 

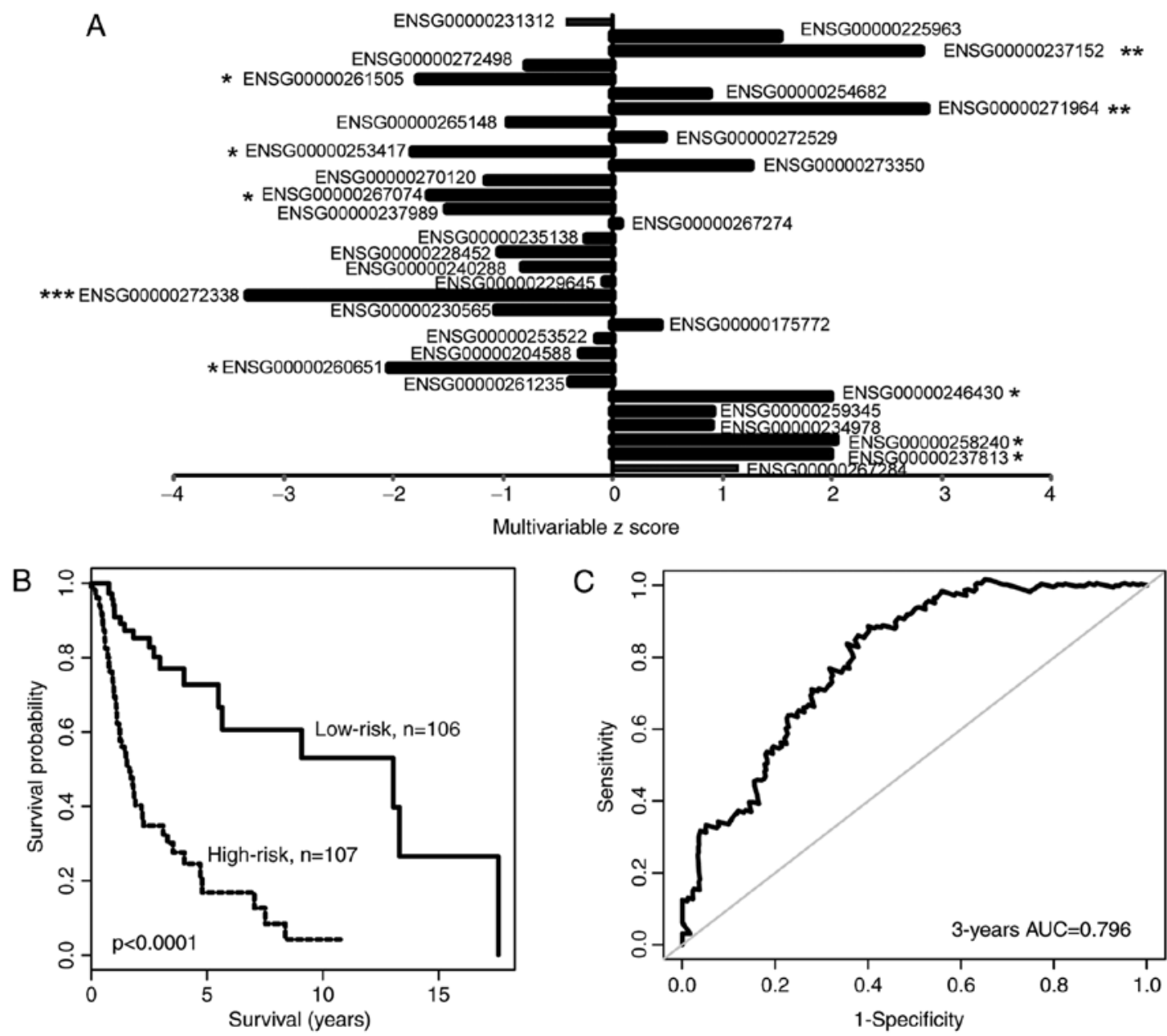

Figure 1. Identification of prognostic lncRNAs in the training cohort. (A) Multivariate analysis of the expression levels of 32 candidate survival-associated IncRNAs, with OS time as the dependent variable. ${ }^{*} \mathrm{P}<0.05,{ }^{* *} \mathrm{P}<0.01$ and ${ }^{* * *} \mathrm{P}<0.001$. (B) Kaplan-Meier survival curves of OS time for the high- and low-risk groups. (C) Receiver operating characteristic curve of the lncRNA expression signature in predicting the 3-year OS time of patients with head and neck squamous cell carcinoma. IncRNAs, long non-coding RNAs; OS, overall survival; AUC, area under the curve.

performance of the IncRNA expression signature, the lncRNA expression signature was validated in the test cohort. By using the aforementioned risk score model, 212 patients from the testing cohort were classified into high-risk $(\mathrm{n}=85)$ and low-risk $(n=127)$ groups using the risk score cut-off derived from training cohort (-1.0376). As observed in the training cohort, the OS time of patients in the high-risk group was significantly shorter compared with that of patients in the low-risk group (median OS time, 2.36 vs. 4.83 years, respectively; $\mathrm{P}=0.0075$; Fig. 2A). In univariate analysis, the HR of the high-risk vs. low-risk group for OS time was 1.907 (95\% CI, 1.179-3.085; $\mathrm{P}=0.0085$; Table II). The 3- and 5-year survival rates of the high-risk group were 43.7 and $39.3 \%$, respectively, whereas those of the low-risk group were 71.9 and $49.9 \%$, respectively. The AUC of the time-dependent ROC curve for the lncRNA expression signature was 0.637 for 3 -year OS time $(\mathrm{P}<0.01$; Fig. 2B).

The prognostic value of the IncRNA signature was subsequently analyzed in the entire TCGA cohort of 425 patients. Using the aforementioned risk score model and cut-off value of the training cohort, patients were segregated into high-risk $(n=179)$ and low-risk $(n=233)$ groups with significantly different OS times (median OS time, 1.79 vs. 9.08 years;
$\mathrm{P}<0.0001$; Fig. 2C). In univariate analysis, the HR of the high-risk vs. low-risk group for OS time was 3.014 (95\% CI, 2.111-4.304; $\mathrm{P}<0.00011$ Table II). The 3- and 5-year survival rates of the high-risk group were 39.1 and $27.1 \%$, respectively, whereas those of the low-risk group were 73.8 and $61.9 \%$, respectively. The AUC of time-dependent ROC curve for the lncRNA expression signature was 0.718 for 3 -year OS time $(\mathrm{P}<0.01$; Fig. 2D).

The distribution of risk score, the survival status of the patients with HNSCC and the expression pattern of the lncRNA biomarkers in the training cohort, testing cohort and entire TCGA cohort are presented in Fig. 3. Patients in the high-risk group exhibited higher expression of the four lncRNAs associated with poor prognosis compared with patients in the low-risk group, whereas patients in the low-risk group expressed higher levels of the six protective prognostic lncRNAs compared with the high-risk group.

IncRNA expression signature is independent of clinicalfeatures. To assess whether the survival-prediction ability of the lncRNA expression signature was independent of clinical features, multivariate Cox regression analysis was performed using the following variables: Risk score, age, sex, anatomic neoplasm 


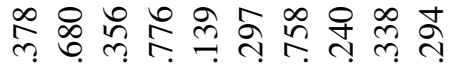

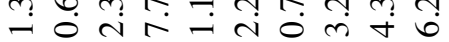

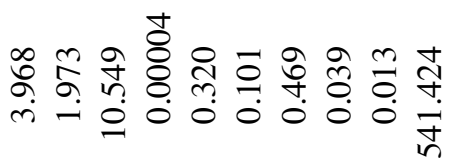

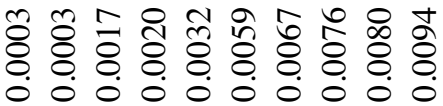

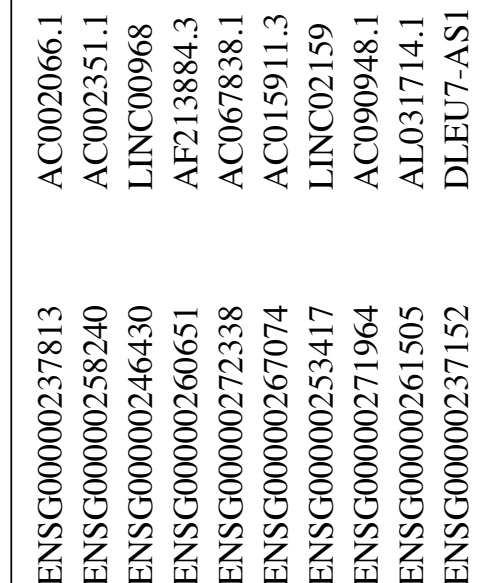

subdivision, history of other malignancies, lymphovascular invasion, perineural invasion, pathological lymph node status $(\mathrm{pN})$, extracapsular spread, clinical stage, pathological stage, alcohol-consumption history, margin status and tumor grade. The results demonstrated that the lncRNA expression signature was significantly associated with OS time in the training cohort (HR, 16.03; 95\% CI, 3.609-71.154; $\mathrm{P}=0.0003$ ), testing cohort (HR, 4.337; 95\% CI, 1.245-15.108; $\mathrm{P}=0.0212$ ) and entire TCGA cohort (HR, 4.5375; 95\% CI, 2.169-9.491; P=0.0001; Table II).

Data stratification analysis was performed for age and $\mathrm{pN}$ status, as these two variables were significant in the multivariate analysis. The patients were divided into two cohorts: Younger $(<60$ years; $n=183)$ and older $(\geq 60$ years; $n=242)$. Using the lncRNA expression signature, patients in the younger cohort were further divided into high-risk and low-risk groups (Fig. 4A). Similar results were observed when the lncRNA expression signature was applied to the older cohort, in which patients were further divided into high-risk and low-risk groups (Fig. 4B). All patients were subsequently divided into two patient cohorts according to $\mathrm{pN}$ status: $\mathrm{pN}$-positive cohort $(n=339)$ and $\mathrm{pN}$-negative cohort $(\mathrm{n}=45)$. The lncRNA expression signature was applied to classify patients of the $\mathrm{pN}$-positive cohort and the pN-negative cohort into high-risk and low-risk groups. Kaplan-Meier survival analysis indicated that the OS time of patients in the high-risk group was significantly shorter compared with that of patients in the low-risk group, despite having the same $\mathrm{pN}$ status $(\mathrm{P}<0.0001$ for $\mathrm{pN}$-positive cohort and $\mathrm{P}=0.0014$ for $\mathrm{pN}$-negative cohort). These results indicated that the predictive ability of the lncRNA expression signature was independent of commonly used clinical features for predicting the survival of patients with HNSCC.

Functional characteristics of the identified IncRNA biomarkers. To investigate the potential biological role of the identified lncRNA biomarkers, GO and KEGG functional enrichment analysis of protein-coding genes associated with the identified IncRNA biomarkers was performed. The correlations between protein-coding genes and the lncRNA biomarkers were identified by Pearson's correlation coefficient using the entire TCGA data cohort of 425 patients with HNSCC. The protein-coding genes ranked in the top $1 \%$ were used for GO and KEGG functional enrichment analysis. The results of GO analysis suggested that the protein-coding genes were enriched in 14 GO terms, which could be categorized into two functional clusters: 'Cell-adhesion' and 'immune response' (Fig. 5A). The results of GO analysis suggested that protein-coding genes correlated with the identified lncRNA biomarkers were enriched in 11 KEGG biological pathways, including 'Cytokine-cytokine receptor interaction', 'Primary immunodeficiency', 'Cell adhesion molecules', 'Hematopoietic cell lineage', 'T cell receptor signaling pathway', 'Ras signaling pathway', 'Focal adhesion', 'Rapl signaling pathway', 'Regulation of actin cytoskeleton', 'Pathways in cancer', and 'PI3K-Akt signaling pathway' (Fig. 5B).

\section{Discussion}

HNSCC is a heterogeneous disease characterized by distinct clinical and molecular features (20). Traditional staging diagnosis, treatment options and prognostic prediction of HNSCC 
Table II. Univariate and multivariate Cox regression analysis of overall survival in each patient cohort.

A, Training cohort $(n=213)$

\begin{tabular}{|c|c|c|c|c|c|c|}
\hline \multirow[b]{2}{*}{ Variable } & \multicolumn{3}{|c|}{ Univariate analysis } & \multicolumn{3}{|c|}{ Multivariate analysis } \\
\hline & HR & $95 \% \mathrm{CI}$ of $\mathrm{HR}$ & P-value & HR & $95 \% \mathrm{CI}$ of $\mathrm{HR}$ & P-value \\
\hline \multicolumn{7}{|l|}{ 10-IncRNA risk score } \\
\hline High/low & 5.1420 & $2.924-9.043$ & $<0.0001$ & 16.0300 & $3.609-71.154$ & 0.0003 \\
\hline \multicolumn{7}{|l|}{ Age, years } \\
\hline$\geq 60 /<60$ & 1.2850 & $0.803-2.058$ & 0.2960 & 1.9340 & $0.718-5.211$ & 0.1921 \\
\hline \multicolumn{7}{|l|}{ Sex } \\
\hline Male/Female & 0.9770 & $0.588-1.622$ & 0.9280 & 1.2660 & $0.39-4.113$ & 0.6945 \\
\hline \multicolumn{7}{|c|}{ Anatomic neoplasm subdivision } \\
\hline Larynx/Others & 1.7810 & $0.934-3.396$ & 0.0798 & 1.8800 & $0.429-8.236$ & 0.4021 \\
\hline Oral cavity/Others & 1.0140 & $0.488-2.108$ & 0.9697 & 0.0801 & $0.007-0.919$ & 0.0426 \\
\hline Oral tongue/Others & 1.2080 & $0.627-2.33$ & 0.5721 & 0.9678 & $0.215-4.353$ & 0.9660 \\
\hline \multicolumn{7}{|c|}{ History of other malignancy } \\
\hline Yes/No & 0.3650 & $0.051-2.638$ & 0.3180 & $<0.0001$ & $0-\infty$ & 0.9982 \\
\hline \multicolumn{6}{|c|}{ Lymphovascular invasion present } & 0.1932 \\
\hline \multicolumn{7}{|c|}{ Perineural invasion present } \\
\hline Yes/No & 1.4150 & $0.754-2.657$ & 0.2800 & 2.1350 & $0.543-8.401$ & 0.2776 \\
\hline \multicolumn{7}{|l|}{$\mathrm{pN}$} \\
\hline Positive/Negative & 0.7470 & $0.379-1.472$ & 0.4000 & 0.0222 & $0.002-0.296$ & 0.0040 \\
\hline \multicolumn{7}{|l|}{ ECS } \\
\hline Positive/Negative & 2.2550 & $1.236-4.114$ & 0.0081 & 2.2020 & $0.593-8.174$ & 0.2384 \\
\hline \multicolumn{7}{|l|}{ Clinical stage } \\
\hline III,IV/I,II & 1.2750 & $0.761-2.136$ & 0.3567 & 2.6400 & $0.459-15.195$ & 0.2769 \\
\hline \multicolumn{6}{|l|}{ Pathological stage } & 0.6918 \\
\hline \multicolumn{7}{|l|}{ Alcohol history } \\
\hline Yes/No & 0.7990 & $0.496-1.289$ & 0.3581 & 4.1760 & $0.955-18.265$ & 0.0576 \\
\hline \multicolumn{7}{|l|}{ Margin status } \\
\hline Positive/Negative & 1.2310 & $0.64-2.365$ & 0.5332 & 8.2830 & $0.954-71.921$ & 0.0552 \\
\hline \multicolumn{7}{|l|}{ Tumor grade } \\
\hline $\mathrm{G} 3,4 / \mathrm{G} 1,2$ & 0.7850 & $0.453-1.359$ & 0.3867 & 3.6370 & $1.178-11.228$ & 0.0247 \\
\hline
\end{tabular}

B, Test cohort $(\mathrm{n}=212)$

\begin{tabular}{|c|c|c|c|c|c|c|}
\hline \multirow[b]{2}{*}{ Variable } & \multicolumn{3}{|c|}{ Univariate analysis } & \multicolumn{3}{|c|}{ Multivariate analysis } \\
\hline & HR & $95 \% \mathrm{CI}$ of $\mathrm{HR}$ & P-value & HR & $95 \% \mathrm{CI}$ of $\mathrm{HR}$ & P-value \\
\hline \multicolumn{7}{|l|}{ 10-lncRNA risk score } \\
\hline High/low & 1.9070 & $1.179-3.085$ & 0.0085 & 4.3370 & $1.245-15.108$ & 0.0212 \\
\hline \multicolumn{7}{|l|}{ Age, years } \\
\hline$\geq 60 /<60$ & 1.2590 & $0.774-2.049$ & 0.3529 & 6.0650 & $1.297-28.365$ & 0.0220 \\
\hline \multicolumn{7}{|l|}{ Sex } \\
\hline Male/Female & 0.7420 & $0.45-1.224$ & 0.2424 & 2.0370 & $0.442-9.386$ & 0.3614 \\
\hline \multicolumn{7}{|c|}{ Anatomic neoplasm subdivision } \\
\hline Larynx/Others & 0.6913 & $0.344-1.388$ & 0.2990 & $<0.0001$ & $0-\infty$ & 0.9971 \\
\hline Oral cavity/Others & 1.3777 & $0.742-2.56$ & 0.3110 & 1.3930 & $0.277-7.009$ & 0.6873 \\
\hline Oral tongue/Others & 1.2292 & $0.631-2.395$ & 0.5440 & 0.3850 & $0.095-1.552$ & 0.1796 \\
\hline
\end{tabular}


Table II. Continued.

B, Test cohort $(\mathrm{n}=212)$

\begin{tabular}{|c|c|c|c|c|c|c|}
\hline \multirow[b]{2}{*}{ Variable } & \multicolumn{3}{|c|}{ Univariate analysis } & \multicolumn{3}{|c|}{ Multivariate analysis } \\
\hline & HR & $95 \% \mathrm{CI}$ of $\mathrm{HR}$ & P-value & HR & $95 \% \mathrm{CI}$ of $\mathrm{HR}$ & P-value \\
\hline \multicolumn{7}{|c|}{ History of other malignancy } \\
\hline Yes/No & 1.0080 & $0.404-2.516$ & 0.9861 & 0.6159 & $0.061-6.203$ & 0.6809 \\
\hline \multicolumn{7}{|c|}{ Lymphovascular invasion present } \\
\hline Yes/No & 1.4140 & $0.746-2.683$ & 0.2885 & 3.6560 & $0.829-16.13$ & 0.0869 \\
\hline \multicolumn{7}{|c|}{ Perineural invasion present } \\
\hline Yes/No & 1.8060 & $0.991-3.291$ & 0.0535 & 4.0110 & $1.049-15.345$ & 0.0424 \\
\hline \multicolumn{7}{|l|}{$\mathrm{pN}$} \\
\hline Positive/Negative & 0.5530 & $0.28-1.094$ & 0.0889 & 0.0018 & $0.0002-0.1168$ & 0.0030 \\
\hline \multicolumn{7}{|l|}{ ECS } \\
\hline Positive/Negative & 1.9730 & $1.105-3.523$ & 0.0216 & 0.7748 & $0.196-3.071$ & 0.7165 \\
\hline \multicolumn{7}{|l|}{ Clinical stage } \\
\hline III,IV/I,II & 0.9490 & $0.54-1.667$ & 0.8554 & 16.1200 & $1.204-215.749$ & 0.0357 \\
\hline \multicolumn{7}{|l|}{ Pathologic stage } \\
\hline III,IV/I,II & 1.2590 & $0.667-2.376$ & 0.4771 & 1.7120 & $0.136-21.539$ & 0.6772 \\
\hline \multicolumn{7}{|l|}{ Alcohol history } \\
\hline Yes/No & 0.9900 & $0.601-1.631$ & 0.9696 & 3.4710 & $0.684-17.617$ & 0.1332 \\
\hline \multicolumn{7}{|l|}{ Margin status } \\
\hline Positive/Negative & 1.8580 & $0.954-3.617$ & 0.0685 & 0.7339 & $0.095-5.688$ & 0.7671 \\
\hline \multicolumn{7}{|l|}{ Tumor grade } \\
\hline $\mathrm{G} 3,4 / \mathrm{G} 1,2$ & 0.8440 & $0.504-1.412$ & 0.5178 & 2.6290 & $0.589-11.728$ & 0.2052 \\
\hline
\end{tabular}

C, Entire The Cancer Genome Atlas cohort $(n=425)$

\begin{tabular}{|c|c|c|c|c|c|c|}
\hline \multirow[b]{2}{*}{ Variable } & \multicolumn{3}{|c|}{ Univariate analysis } & \multicolumn{3}{|c|}{ Multivariate analysis } \\
\hline & HR & $95 \% \mathrm{CI}$ of $\mathrm{HR}$ & P-value & HR & $95 \% \mathrm{CI}$ of $\mathrm{HR}$ & P-value \\
\hline \multicolumn{7}{|l|}{ 10-lncRNA risk score } \\
\hline High/low & 3.0140 & 2.111-4.304 & $<0.0001$ & 4.5375 & $2.169-9.491$ & 0.0001 \\
\hline \multicolumn{7}{|l|}{ Age, years } \\
\hline$\geq 60 /<60$ & 1.2860 & $0.918-1.802$ & 0.1440 & 1.8743 & $0.947-3.709$ & 0.0712 \\
\hline \multicolumn{7}{|l|}{ Sex } \\
\hline Male/Female & 0.8370 & $0.588-1.192$ & 0.3250 & 1.1553 & $0.519-2.573$ & 0.7239 \\
\hline \multicolumn{7}{|c|}{ Anatomic neoplasm subdivision } \\
\hline Larynx/Others & 1.1600 & $0.733-1.836$ & 0.5270 & 0.3415 & $0.105-1.112$ & 0.0745 \\
\hline Oral cavity/Others & 1.2170 & $0.762-1.944$ & 0.4120 & 0.5533 & $0.178-1.719$ & 0.3062 \\
\hline Oral tongue/Others & 1.1780 & $0.745-1.864$ & 0.4840 & 0.9037 & $0.418-1.956$ & 0.7972 \\
\hline \multicolumn{7}{|c|}{ History of other malignancy } \\
\hline Yes/No & 0.7650 & $0.337-1.737$ & 0.5216 & 0.5668 & $0.069-4.659$ & 0.5973 \\
\hline \multicolumn{7}{|c|}{ Lymphovascular invasion present } \\
\hline Yes/No & 1.3320 & $0.844-2.1$ & 0.2183 & 1.0942 & $0.512-2.339$ & 0.8165 \\
\hline \multicolumn{7}{|c|}{ Perineural invasion present } \\
\hline Yes/No & 1.6360 & $1.06-2.526$ & 0.0263 & 1.8780 & $0.894-3.947$ & 0.0963 \\
\hline \multicolumn{7}{|l|}{$\mathrm{pN}$} \\
\hline Positive/Negative & 0.6400 & $0.396-1.034$ & 0.0680 & 0.0264 & $0.003-0.2$ & 0.0004 \\
\hline \multicolumn{7}{|l|}{$\mathrm{ECS}$} \\
\hline Positive/Negative & 2.0310 & $1.342-3.074$ & 0.0008 & 1.4968 & $0.692-3.237$ & 0.3055 \\
\hline
\end{tabular}


Table II. Continued.

C, Entire The Cancer Genome Atlas cohort $(n=425)$

\begin{tabular}{|c|c|c|c|c|c|c|}
\hline \multirow[b]{2}{*}{ Variable } & \multicolumn{3}{|c|}{ Univariate analysis } & \multicolumn{3}{|c|}{ Multivariate analysis } \\
\hline & HR & $95 \% \mathrm{CI}$ of $\mathrm{HR}$ & P-value & HR & $95 \% \mathrm{CI}$ of HR & P-value \\
\hline \multicolumn{7}{|l|}{ Clinical stage } \\
\hline III,IV/I,II & 1.1240 & $0.771-1.638$ & 0.5433 & 2.7613 & $0.729-10.464$ & 0.1351 \\
\hline \multicolumn{7}{|l|}{ Pathological stage } \\
\hline III,IV/I,II & 1.4940 & $0.969-2.305$ & 0.0694 & 1.0189 & $0.278-3.732$ & 0.9775 \\
\hline \multicolumn{7}{|l|}{ Alcohol history } \\
\hline Yes/No & 0.8930 & $0.633-1.258$ & 0.5160 & 1.9261 & $0.854-4.342$ & 0.1140 \\
\hline \multicolumn{7}{|l|}{ Margin status } \\
\hline Positive/Negative & 1.5120 & $0.95-2.406$ & 0.0809 & 1.9081 & $0.59-6.173$ & 0.2808 \\
\hline \multicolumn{7}{|l|}{ Tumor grade } \\
\hline $\mathrm{G} 3,4 / \mathrm{G} 1,2$ & 0.8280 & $0.571-1.199$ & 0.3174 & 1.0951 & $0.511-2.346$ & 0.8153 \\
\hline
\end{tabular}

HR, hazard ratio; CI, confidence interval; pN, pathological lymph node status; ECS, extracapsular spread; G, grade.
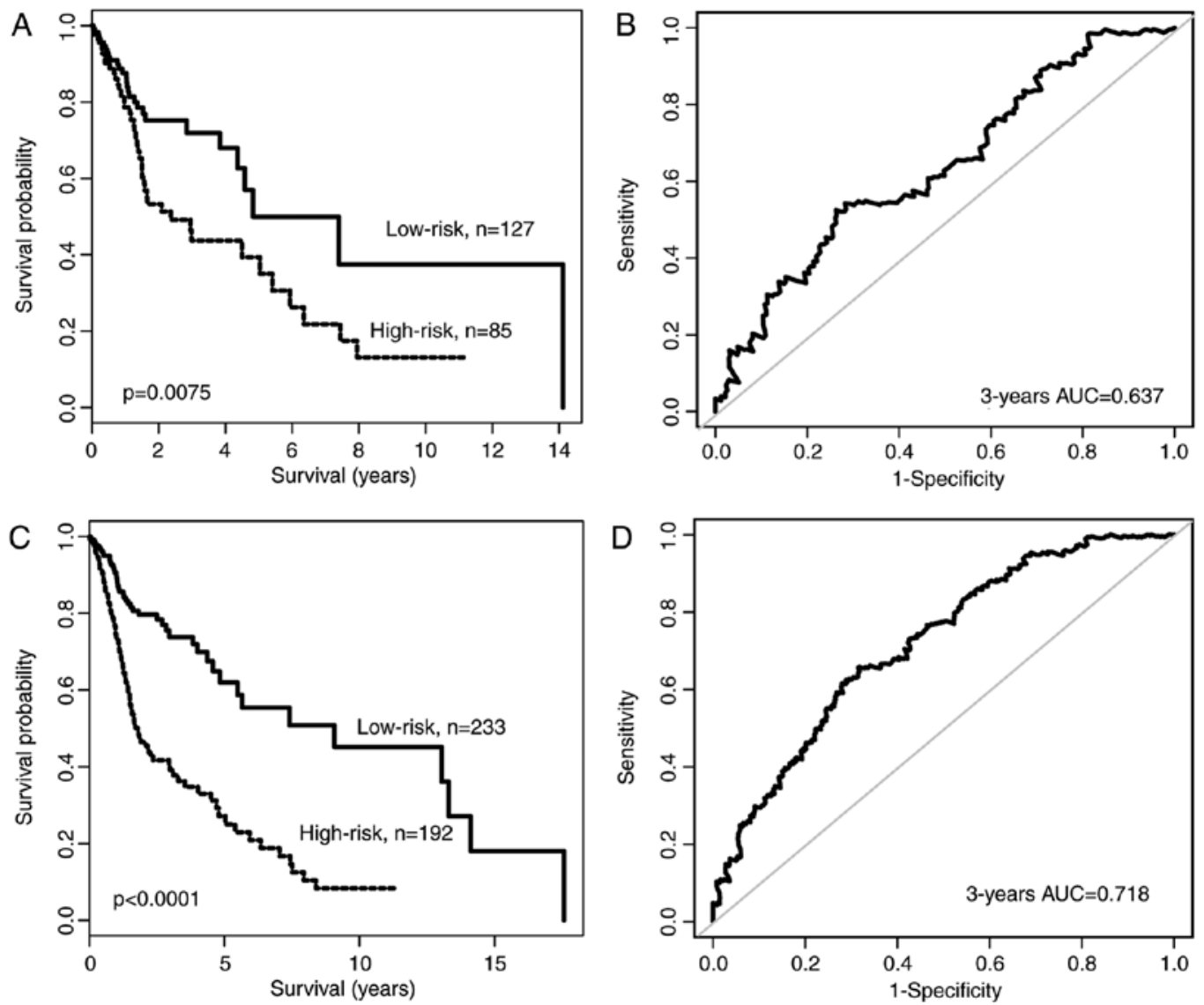

Figure 2. Further validation of the lncRNA expression signature in predicting overall survival. (A) Kaplan-Meier survival curves of the OS time in the high- and low-risk groups in the testing cohort. (B) ROC curve of the lncRNA signature in predicting 3-year OS time of patients in the test cohort. (C) Kaplan-Meier survival curves of OS time between high- and low-groups in the entire TCGA cohort. (D) ROC curve of the IncRNA signature in predicting the 3-year OS time of patients in the entire TCGA cohort. IncRNA, long non-coding RNA; ROC, receiver operating characteristic; AUC, area under the curve; TCGA, The Cancer Genome Atlas.

do not allow for precision medicine, due to the diverse molecular features between patients with identical American Joint Committee on Cancer TNM staging (21). Molecular profiles of patients with HNSCC have been investigated in previous reports, which demonstrated the potential of molecular profiles as novel biomarkers to predict treatment outcome and to 

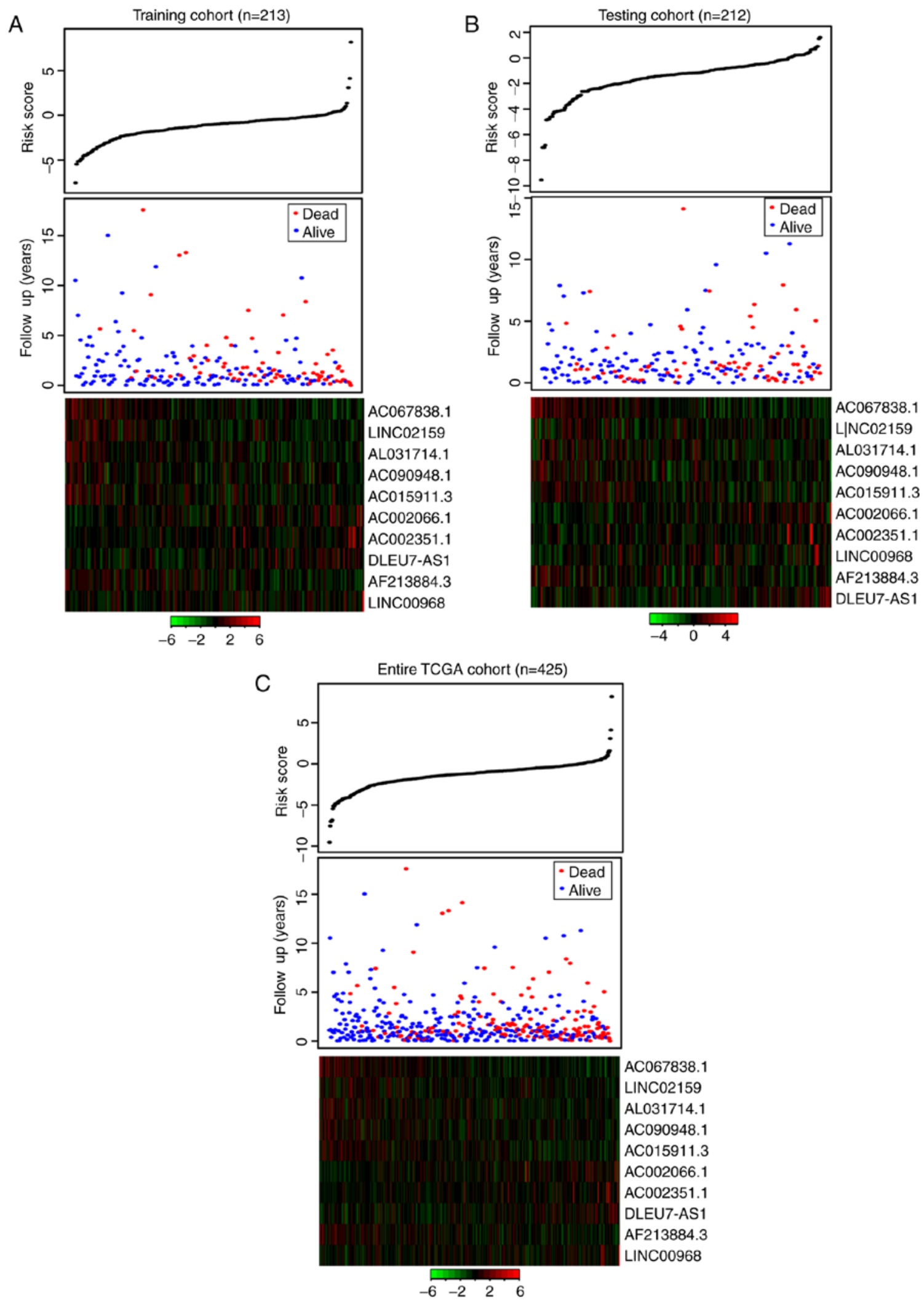

Figure 3. Presentation of the lncRNA signature-based risk scoring of patients with HNSCC. The distribution of risk score, the survival status of patients with HNSCC and the expression pattern of the IncRNA biomarkers in (A) the training cohort, (B) the testing cohort and (C) the entire TCGA cohort. Red represents upregulated lncRNAs and green represents downregulated lncRNAs. IncRNA, long non-coding RNA; HNSCC, head and neck squamous cell carcinoma; TCGA, The Cancer Genome Atlas.

guide treatment strategies (21-24). However, these studies are restricted to protein-coding gene data and miRNA data. An improved understanding of the role of lncRNAs in HNSCC may result in lncRNA expression emerging as a promising molecular biomarker for predicting the prognosis of patients with HNSCC $(25,26)$. 

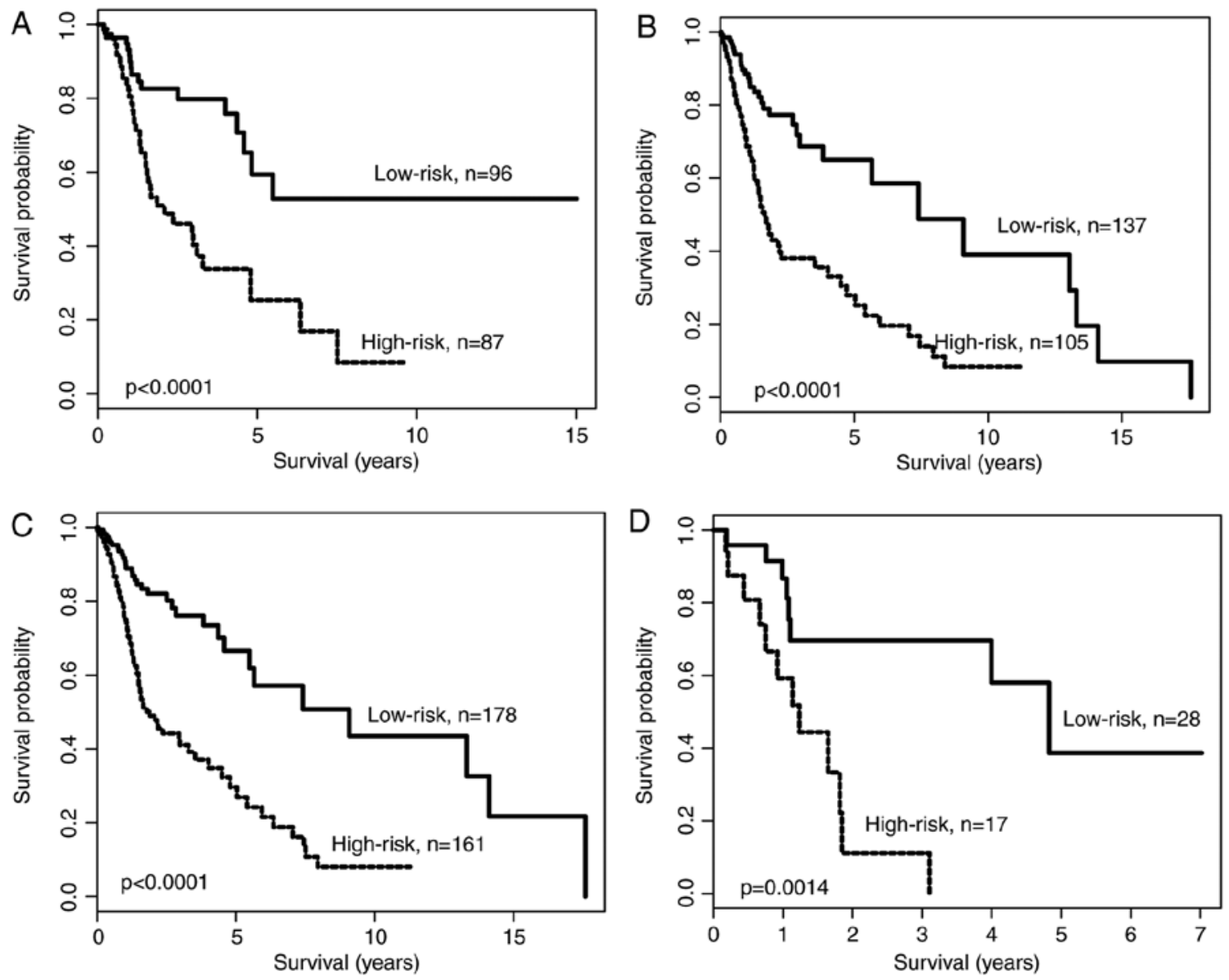

Figure 4. Predictive performance of the long non-coding RNA expression signature is independent of age and pathological lymph node status. (A) Kaplan-Meier survival curves for younger patients with HNSCC ( $<60$ years). (B) Kaplan-Meier survival curves for older patients with HNSCC ( $\geq 60$ years). (C) Kaplan-Meier survival curves for patients with HNSCC with positive pathological lymph node status. (D) Kaplan-Meier survival curves for patients with HNSCC with negative pathological lymph node status. HNSCC, head and neck squamous cell carcinoma.

The current study investigated the prognostic values of IncRNA expression profiles in predicting the OS time of patients with HNSCC by integrating clinical and profiling data from TCGA. A total of ten novel lncRNAs were identified as potential prognostic markers for patients with HNSCC. These were used to develop a prognostic signature using a risk scoring method, which classified the patients into 2 groups with significantly different OS times. The lncRNA signature identified in the training cohort demonstrated a similar prognostic value in the testing and the entire TCGA cohorts. Multivariable Cox regression analysis indicated that the signature was an independent prognostic factor for patients with HSNCC. Thus, the prognostic value of the IncRNA signature may have clinical potential for patients with HNSCC.

Furthermore, functional enrichment analysis was performed to investigate the biological roles of the IncRNA signature in HNSCC. Protein-coding genes, whose expression values were positively associated with the lncRNA signature, were enriched in 14 GO biological terms and 11 KEGG biological pathways. These enriched GO biological processes and KEGG pathways were categorized into 'cell-adhesion' and 'immune response'. Thus, the ten IncRNAs associated with the survival of patients with HNSCC may be involved in cell-adhesion and the immune response. A number of studies have indicated that dysfunction of cell-adhesion and cell-migration serves an important role in the processes of invasion and metastasis in HNSCC $(27,28)$. HNSCC is an immunosuppressive disease characterized by dysregulation of immunocompetent cells and impaired cytokine secretion (29). The immune system serves an important role in the occurrence and progression of HNSCC, and the status of the immune system is likely to be of prognostic value in HNSCC (30). The pathway 'immune response' was significantly associated with the lncRNA signature, suggesting that the lncRNA expression-based risk scoring system described in the current study may reflect the basic status of the immune response in the tumor microenvironment. However, several limitations of the present study should be noted. Firstly, the ten lncRNA biomarkers identified in the present study were only validated in TCGA datasets. Further testing in other independent datasets is required. Secondly, the functions of the ten lncRNA biomarkers were only predicted using bioinformatics methods; therefore, these require further investigation using experimental methods.

In conclusion, the present study identified ten IncRNAs associated with the OS time of patients with HSNCC from a large cohort. These ten IncRNA biomarkers were used to develop a lncRNA signature which robustly predicted the survival of patients with HSNCC in the training, testing and entire TCGA 
A

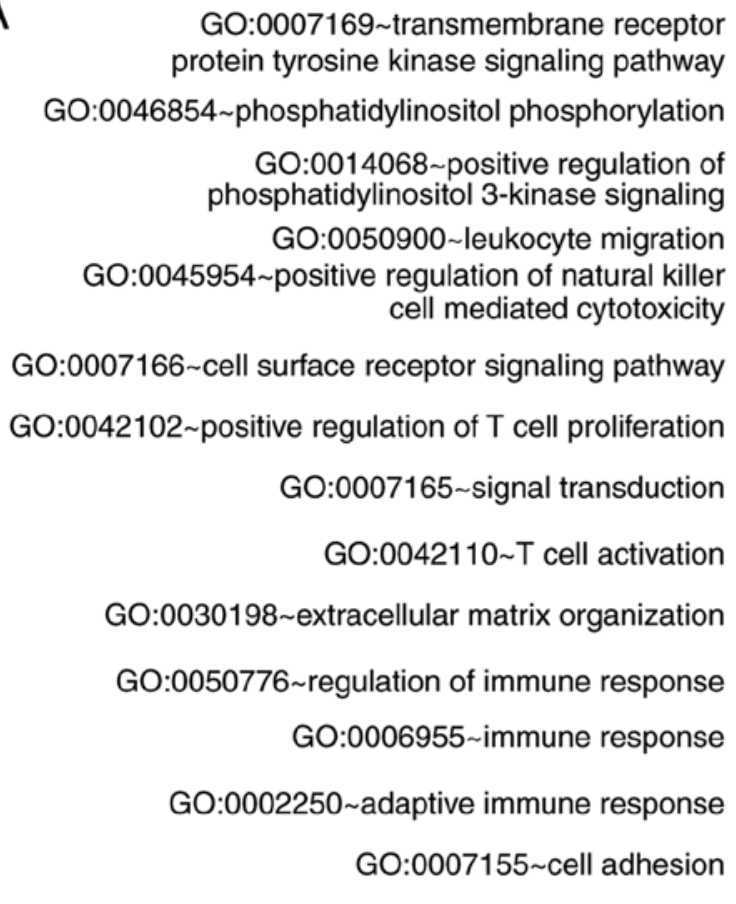

GO:0043547 positive regulation of GTPase activity

\section{B}

hsa04151:PI3K-Akt signaling pathway hsa05200:Pathways in cancer hsa04810:Regulation of actin cytoskeleton hsa04015:Rap1 signaling pathway hsa04510:Focal adhesion hsa04014:Ras signaling pathway hsa04660:T cell receptor signaling pathway hsa04640:Hematopoietic cell lineage hsa04514:Cell adhesion molecules (CAMs) hsa05340:Primary immunodeficiency hsa04060:Cytokine-cytokine receptor interaction

\section{(n) \\ 1
0}

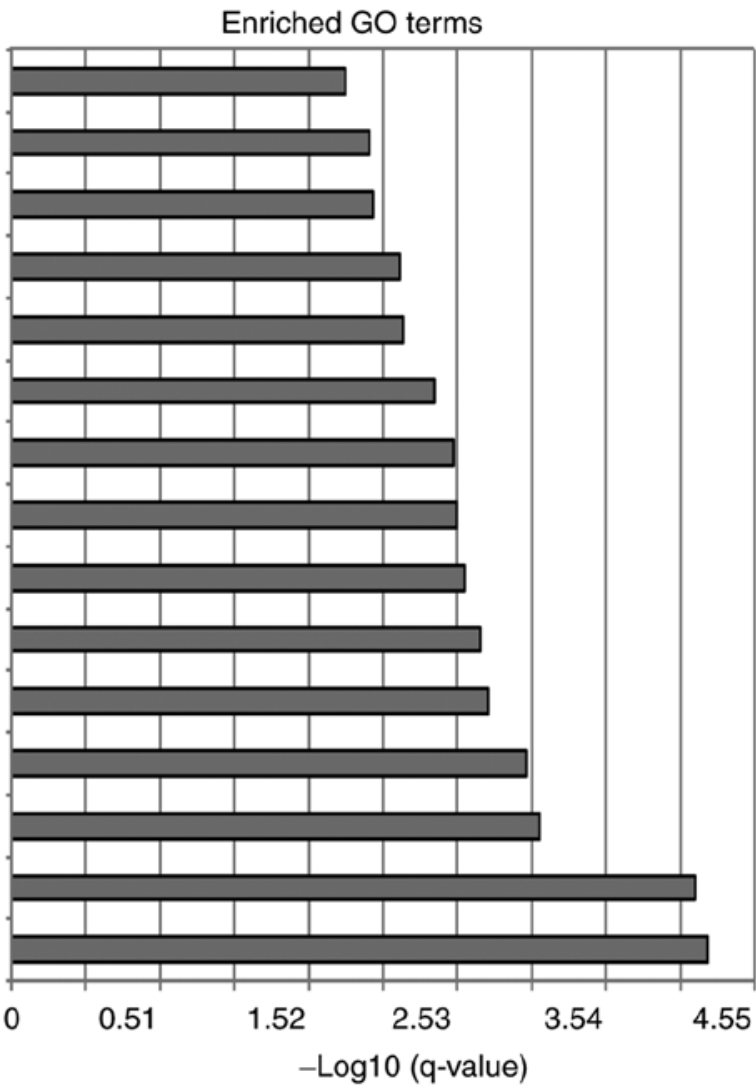

Enriched KEGG pathways

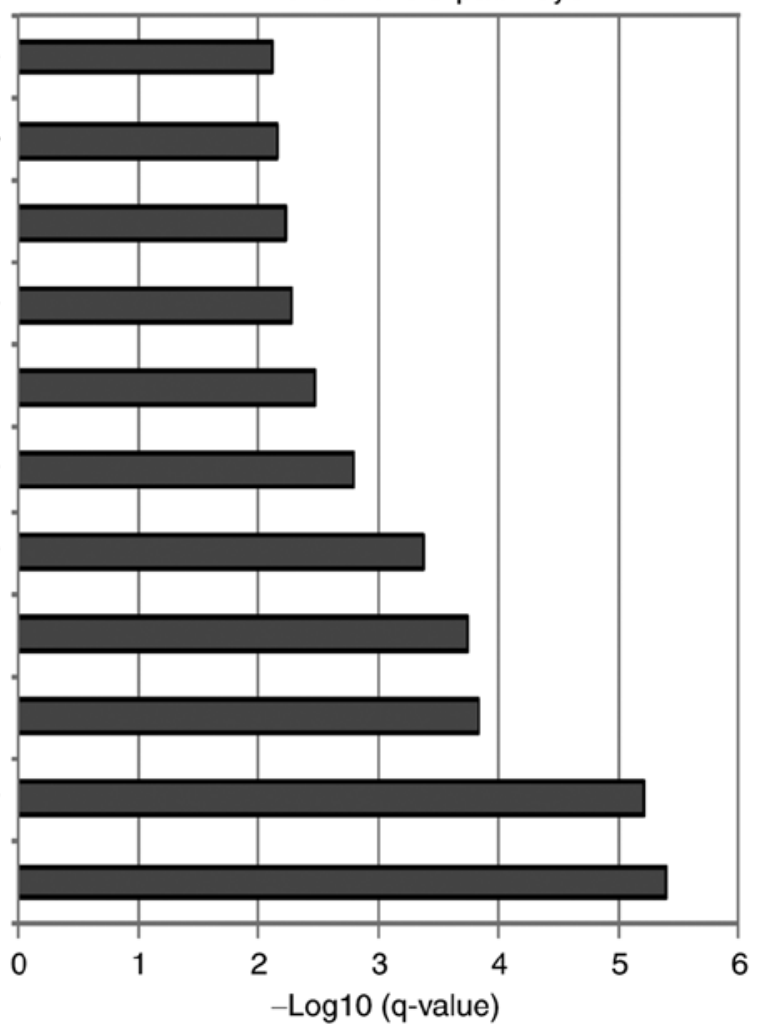

Figure 5. Functional annotations of the long non-coding RNA signature. (A) Enriched GO terms. (B) Enriched KEGG pathways. GO, Gene Ontology; KEGG, Kyoto Encyclopedia of Genes and Genomes.

cohorts. Further analysis revealed that the prognostic value was independent of the clinical and pathological characteristics of patients with HSNCC. While the results presented in the current study require further validation, the current study indicates that lncRNA expression profiles may be used as molecular markers to improve the clinical prognosis for patients with HSNCC. 


\section{Acknowledgements}

Not applicable.

\section{Funding}

The present study was supported by Hubei Province Health and Family Planning Scientific Research Project (grant no. WJ2017M145).

\section{Availability of data and material}

The datasets generated and/or analyzed during the current study are available in the The Cancer Genome Atlas (TCGA; tcga-data. nci.nih.gov/) and The Atlas of Noncoding RNAs in Cancer (bioinformatics.mdanderson.org/main/TANRIC:Overview).

\section{Authors' contributions}

YL conceived and designed the experiments. JL, YHL, XW performed the experiments and analyzed the data. YL wrote the paper. All authors read and approved the final manuscript.

\section{Ethics approval and consent to participate}

Not applicable.

\section{Patient consent for publication}

Not applicable.

\section{Competing interests}

The authors declare that they have no competing interests.

\section{References}

1. Feng Z, Xu QS, Qin LZ, Li H, Huang X, Su M and Han Z: Second primary cancer after index head and neck squamous cell carcinoma in Northern China. Oral Surg Oral Med Oral Pathol Oral Radiol 123: 95-102, 2017.

2. Pulte D and Brenner H: Changes in survival in head and neck cancers in the late 20th and early 21 st century: A period analysis. Oncologist 15: 994-1001, 2010.

3. Grégoire V, Lefebvre JL, Licitra L and Felip E; EHNS-ESMO-ESTRO Guidelines Working Group: Squamous cell carcinoma of the head and neck: EHNS-ESMO-ESTRO clinical practice guidelines for diagnosis, treatment and follow-up. Ann Oncol 21 (Suppl 5): v184-v186, 2010.

4. ENCODE Project Consortium,Birney E,Stamatoyannopoulos JA, Dutta A, Guigó R, Gingeras TR, Margulies EH, Weng Z, Snyder M, Dermitzakis ET, et al: Identification and analysis of functional elements in $1 \%$ of the human genome by the ENCODE pilot project. Nature 447: 799-816, 2007.

5. Hangauer MJ, Vaughn IW and McManus MT: Pervasive transcription of the human genome produces thousands of previously unidentified long intergenic noncoding RNAs. PLoS Genet 9: e1003569, 2013.

6. Zhang H, Chen Z, Wang X, Huang Z, He Z and Chen Y: Long non-coding RNA: A new player in cancer. J Hematol Oncol 6: 37, 2013.

7. Zhou M, Zhang Z, Zhao H, Bao S, Cheng L and Sun J: An immune-related six-lncRNA signature to improve prognosis prediction of glioblastoma multiforme. Mol Neurobiol 55: 3684-3697, 2018

8. Zhou M, Zhao H, Xu W, Bao S, Cheng L and Sun J: Discovery and validation of immune-associated long non-coding RNA biomarkers associated with clinically molecular subtype and prog nosis in diffuse large B cell lymphoma. Mol Cancer 16: 16, 2017.
9. Zhou M, Zhang Z, Zhao H, Bao S and Sun J: A novel lncRNA-focus expression signature for survival prediction in endometrial carcinoma. BMC Cancer 18: 39, 2018.

10. Zhou M, Zhao H, Wang X, Sun J and Su J: Analysis of long noncoding RNAs highlights region-specific altered expression patterns and diagnostic roles in Alzheimer's disease. Brief Bioinform Apr 17, 2018. Doi: 10.1093/bib/bby021.

11. Guan GF, Zhang DJ, Wen LJ, Xin D, Liu Y, Yu DJ, Su K, Zhu L, Guo YY and Wang K: Overexpression of lncRNA H19/miR-675 promotes tumorigenesis in head and neck squamous cell carcinoma. Int J Med Sci 13: 914-922, 2016.

12. Wu B, Liu J, Wang B, Liao X, Cui Z and Ding N: Association on polymorphisms in LncRNA HOTAIR and susceptibility to HNSCC in Chinese population. Eur Rev Med Pharmacol Sci 22: 702-706, 2018.

13. Yu J, Liu Y, Guo C, Zhang S, Gong Z, Tang Y, Yang L, He Y, Lian Y, Li X, et al: Upregulated long non-coding RNA LINC00152 expression is associated with progression and poor prognosis of tongue squamous cell carcinoma. J Cancer 8: 523-530, 2017.

14. Li J, Han L, Roebuck P, Diao L, Liu L, Yuan Y, Weinstein JN and Liang H: TANRIC: An interactive open platform to explore the function of lncRNAs in cancer. Cancer Res 75: 3728-3737, 2015.

15. Zhou M, Guo M, He D, Wang X, Cui Y, Yang H, Hao D and Sun J: A potential signature of eight long non-coding RNAs predicts survival in patients with non-small cell lung cancer. J Transl Med 13: 231, 2015.

16. Zhou M, Sun Y, Sun Y, Xu W, Zhang Z,Zhao H, Zhong Z and Sun J: Comprehensive analysis of IncRNA expression profiles reveals a novel lncRNA signature to discriminate nonequivalent outcomes in patients with ovarian cancer. Oncotarget 7: 32433-32448, 2016.

17. Zhou M, Zhao H, Wang Z, Cheng L, Yang L, Shi H, Yang H and Sun J: Identification and validation of potential prognostic IncRNA biomarkers for predicting survival in patients with multiple myeloma. J Exp Clin Cancer Res 34: 102, 2015.

18. Blanche P, Dartigues JF and Jacqmin-Gadda H: Estimating and comparing time-dependent areas under receiver operating characteristic curves for censored event times with competing risks. Stat Med 32: 5381-5397, 2013.

19. Moreno-Betancur M, Sadaoui H, Piffaretti C and Rey G: Survival analysis with multiple causes of death: Extending the competing risks model. Epidemiology 28: 12-19, 2017.

20. Cancer Genome Atlas Network: Comprehensive genomic characterization of head and neck squamous cell carcinomas. Nature 517: 576-582, 2015.

21. Chung CH, Parker JS, Karaca G, Wu J, Funkhouser WK, Moore D, Butterfoss D, Xiang D, Zanation A, Yin X, et al: Molecular classification of head and neck squamous cell carcinomas using patterns of gene expression. Cancer Cell 5: 489-500, 2004.

22. Feldman R, Gatalica Z, Knezetic J, Reddy S, Nathan CA, Javadi N and Teknos T: Molecular profiling of head and neck squamous cell carcinoma. Head Neck 38 (Suppl 1): E1625-E1638, 2016.

23. Ramdas L, Giri U, Ashorn CL, Coombes KR, El-Naggar A, Ang KK and Story MD: miRNA expression profiles in head and neck squamous cell carcinoma and adjacent normal tissue. Head Neck 31: 642-654, 2009.

24. Hui AB, Lenarduzzi M, Krushel T, Waldron L, Pintilie M, Shi W, Perez-Ordonez B, Jurisica I, O'Sullivan B, Waldron J, et al: Comprehensive MicroRNA profiling for head and neck squamous cell carcinomas. Clin Cancer Res 16: 1129-1139, 2010.

25. Cao W, Liu JN, Liu Z, Wang X, Han ZG, Ji T, Chen WT and Zou X: A three-lncRNA signature derived from the Atlas of ncRNA in cancer (TANRIC) database predicts the survival of patients with head and neck squamous cell carcinoma. Oral Oncol 65: 94-101, 2017.

26. Zhang ZL, Zhao LJ, Chai L, Zhou SH, Wang F, Wei Y, Xu YP and Zhao P: Seven LncRNA-mRNA based risk score predicts the survival of head and neck squamous cell carcinoma. Sci Rep 7: 309, 2017.

27. Behrens J: The role of cell adhesion molecules in cancer invasion and metastasis. Breast Cancer Res Treat 24: 175-184, 1993.

28. Howell GM and Grandis JR: Molecular mediators of metastasis in head and neck squamous cell carcinoma. Head Neck 27: 710-717, 2005.

29. Varilla V, Atienza J and Dasanu CA: Immune alterations and immunotherapy prospects in head and neck cancer. Expert Opin Biol Ther 13: 1241-1256, 2013.

30. Economopoulou P, Perisanidis C, Giotakis EI and Psyrri A: The emerging role of immunotherapy in head and neck squamous cell carcinoma (HNSCC): Anti-tumor immunity and clinical applications. Ann Transl Med 4: 173, 2016.

This work is licensed under a Creative Commons

Attribution-NonCommercial-NoDerivatives 4.0 International (CC BY-NC-ND 4.0) License. 